\title{
A pilot study evaluating serum pro-prostate-specific antigen in patients with rising PSA following radical prostatectomy
}

\author{
ANTONINO SOTTILE ${ }^{1}$, CINZIA ORTEGA ${ }^{2}$, ALFREDO BERRUTI $^{3}$, MONICA MANGIONI $^{1}$, \\ SARA SAPONARO ${ }^{1}$, ALESSANDRA POLO $^{1}$, VERONICA PRATI $^{2}$, GIOVANNI MUTO $^{4}$, \\ MASSIMO AGLIETTA $^{2}$ and FILIPPO MONTEMURRO ${ }^{2}$
}

\author{
${ }^{1}$ Oncology Foundation of Piedmont, Institute for Cancer Research and Treatment, Laboratory Medicine, Candiolo; \\ ${ }^{2}$ Oncology Foundation of Piedmont, Institute for Cancer Research and Treatment, Medical Oncology 1, Candiolo, Turin; \\ ${ }^{3}$ Department of Clinical and Biological Sciences, Medical Oncology San Luigi Gonzaga Hospital, Orbassano; \\ ${ }^{4}$ Giovanni Bosco Hospital, Division of Urology, Turin, Italy
}

Received September 19, 2011; Accepted December 16, 2011

DOI: 10.3892/ol.2012.570

\begin{abstract}
PSA), a proform of PSA, is a new marker in patients at risk of prostate cancer. We explored the potential role of 2pPSA in the identification of patients with metastatic progression following radical prostatectomy for prostate cancer. Seventy-six patients with biochemical (PSA) recurrence following radical prostatectomy were studied retrospectively. Diagnostic imaging performed at the time of biochemical recurrence confirmed metastatic disease in 31 of the 76 patients. Serum samples were collected and stored at the time of imaging-confirmed metastatic progression or at the most recent procedure for patients with negative imaging. Median values of PSA, free PSA (fPSA), \%fPSA, 2pPSA and prostate health index (PHI) were compared between metastatic and non-metastatic patients by the Mann-Whitney U test. The results of each test were then correlated with metastatic status by univariate and multivariate logistic regression analysis. PSA, fPSA, \%fPSA, 2pPSA serum concentrations and PHI values were statistically significantly higher in patients with metastatic disease. Results of the multivariate analysis revealed that 2 pPSA remained a statistically significant predictor of imaging-proven metastatic prostate cancer among patients with biochemical recurrence. At a cut-off value of $12.25 \mathrm{pg} / \mathrm{ml}, 2 \mathrm{pPSA}$ outperformed the other markers in terms of sensitivity and specificity (97 and $80 \%$, respectively) with respect to imaging-confirmed metastatic progression. This is the first study suggesting that 2pPSA predicts diagnostic imaging-proven metastatic disease in
\end{abstract}

Correspondence to: Dr Filippo Montemurro, Fondazione del Piemonte per l'Oncologia/IRCC Candiolo, Division of Medical Oncology 1, Candiolo, Turin, Italy

E-mail: filippo.montemurro@ircc.it

Key words: prostate cancer, cancer metastasis, biochemical recurrence, [-2]pro-prostate-specific antigen previously resected prostate cancer patients with biochemical recurrence. Our results merit validation in a prospective study.

\section{Introduction}

Prostate-specific antigen (PSA) is the serum marker commonly used for the early detection and prognostication of prostate cancer and is pivotal in the management of this disease (1). In males with an intact prostate, a rising PSA level may be observed in benign conditions such as prostatic hypertrophy or prostatitis and in prostate cancer. Conversely, a rising PSA level following radical prostatectomy or radiation therapy is a state that, if left untreated, is likely to lead to metastasis and mortality from disease (1). The time between rising PSA levels and clinical progression, however, is long on average and not every patient with PSA progression succumbs to the disease (2).

Although a rising PSA level usually constitutes an indication for diagnostic imaging modalities to detect local relapse or metastatic spread, the optimal timing of diagnostic imaging based on rising PSA values is not well defined $(3,4)$. Several PSA cut-off values have been suggested and nomograms including PSA together with other variables have been developed to minimize the number of unnecessary diagnostic procedures (5).

Efforts have also been concentrated on establishing more sensitive and specific alternative PSA-based tests to be used for screening programs, staging of patients with newly diagnosed disease and detection of recurrence.

Examination of the free (f) PSA fraction has led to the identification of different precursor isoforms. One of these isoforms, pro-PSA, may be found in the circulation either in its native form or in various truncated forms containing seven, five or fewer amino acids in the pro-leader peptide (6-8).

Two studies have demonstrated that the [-2]pro-PSA (2pPSA) truncated form is present in tumor extracts and that its serum values are markedly correlated with the presence of prostate cancer. A specific immunoassay has been developed by Beckman Coulter to measure levels of 2pPSA in serum $(9,10)$. In patients with biopsy-confirmed prostate cancer, 2pPSA levels in the $>2$ to $<10 \mathrm{ng} / \mathrm{ml}$ PSA range have 
been shown to improve the specificity for cancer detection $(8,9,11,12)$. The potential role of 2 pPSA in the detection of prostate cancer in healthy subjects has recently been assessed in the context of prospective studies. Sokoll et al have demonstrated that the ratio of 2pPSA to fPSA (\%2pPSA) is capable of improving prostate cancer detection and is correlated with the risk of aggressive disease (13). Le et al have shown that the use of $\% 2$ pPSA or the combination of 2pPSA, PSA and fPSA in the so-called 'prostate health index' (PHI) proved superior in discriminating between prostate cancer and benign disease in males with a total PSA of $2.5-10 \mathrm{ng} / \mathrm{ml}$ and negative digital rectal examination (14). These results have been confirmed in a recent multicenter study conducted in 892 subjects with PSA values between 2 and $10 \mathrm{ng} / \mathrm{ml}$ (15). The use of PHI significantly increased the specificity compared with \%fPSA for prostate cancer detection in males with PSA levels of 2-10 $\mathrm{ng} / \mathrm{ml}$ and a negative digital rectal examination.

Although 2pPSA appears promising as a screening test, it also has a potential usefulness in the detection of recurrent disease, which is unexplored at present. Therefore, we conducted a pilot study in patients who had previously been submitted to prostatectomy and underwent regular follow-up procedures including serial PSA monitoring and diagnostic imaging when required. A comparison of PSA, fPSA, \% fPSA, 2pPSA and PHI values was made in patients with or without imaging-confirmed recurrent prostate cancer. Additionally, we evaluated the potential clinical utility of 2pPSA and PHI in identifying patients with rising PSA levels at a higher risk of progressive disease following prostatectomy.

\section{Materials and methods}

Patients. Subjects undergoing regular follow-up visits following radical prostatectomy due to a histologically confirmed diagnosis of prostate cancer were screened for the purpose of this study. Serial monitoring of serum total PSA was part of routine follow-up according to institutional guidelines. The patients with biochemical recurrence, defined as post-surgical serum PSA $>0.2 \mathrm{ng} / \mathrm{ml}$ with a second confirmatory level of PSA $>0.2 \mathrm{ng} / \mathrm{ml}$ (16), and who were prescribed a computed tomography (CT) scan of the chest and the abdomen and a bone scan to detect metastatic disease were selected for this analysis. Patients submitted to additional imaging modalities including magnetic resonance imaging (MRI) and $18 \mathrm{~F}$-choline positron emission tomography (PET) were also eligible for this study. Of all the PSA-based tests described in the subsequent section, only PSA was available to the treating physician at each follow-up visit. The study followed principles in the Declaration of Helsinki and was approved by the Internal Review Board of the Institute for Cancer Research and Treatment.

Measurement of PSA, fPSA, \%fPSA, 2pPSA serum concentrations and PHI values. Serum samples were analyzed for PSA, fPSA and 2pPSA with the Access 2 Immunoassay System on a UniCel DxI800 instrument (Beckman Coulter, Brea, CA, USA).

The PSA assays were all dual monoclonal sandwich assays using Hybritech antibodies and a chemiluminescent detection system.
The assays for PSA, fPSA and 2pPSA are commercially available. In addition, \%fPSA (fPSA/PSA x 100) and the Beckman Coulter PHI (2pPSA/fPSA) x PSA ${ }^{0.5}$ were calculated.

The 2pPSA assay was calibrated using recombinant 2pPSA with a 7-point calibration curve $(0-5,000 \mathrm{pg} / \mathrm{ml})$ that is stable for at least 28 days. The Limit of Blank and the Limit of Quantization (according to the CLSI guidance document EP17-A, Clinical Laboratory Standards Institute) were 0.5 and $3.23 \mathrm{pg} / \mathrm{ml}$.

Assays were performed from samples collected in BD Vacutainer ${ }^{\circledR}$ serum tubes with gel separator and using the same batch of calibrators.

PSA and fPSA results were obtained in single determinations, 2pPSA in duplicate determination. The analysis was repeated in case of a coefficient of variation $>20 \%$.

Statistical analysis. For each patient, serum samples were collected and used to assess PSA, fPSA, \%fPSA, 2pPSA and PHI values. The samples were stored at the time of the diagnosis of metastatic progression or at the most recent diagnostic imaging procedure in patients for whom metastatic progression was not confirmed.

Patient characteristics were compared using the Chi-square test (categorical variables) and the Mann-Whitney U test (continuous variables). Time to biochemical recurrence was calculated by the Kaplan-Meier method and curves were compared using the log-rank test.

PSA, fPSA, \%fPSA, 2pPSA and PHI values did not follow a normal distribution, even after logarithmic transformation. Therefore, medians and ranges were used as summary statistics and non-parametric tests for comparisons.

Median values of each test were compared between progressing and non-progressing patients by the Mann-Whitney U test. Furthermore, we used receiver operating characteristics (ROC) curves to identify cut-off values discriminating between metastatic and non-metastatic patients for each test with the best combination of sensitivity and specificity. Logistic regression analysis was used to assess the independent value of each test in discriminating progressing from non-progressing patients. The results of each test were studied as continuous and dichotomous variables using cut-off values identified by the ROC curves. In the multivariate analyses, the tests were included in the initial step and removed by backward elimination if their $\mathrm{P}$-value calculated by the Wald statistic was $\geq 0.05$. Univariate and multivariate odds ratios were corrected by $\mathrm{pT}$ and Gleason score at initial diagnosis. Multicollinearity was initially checked by identifying large bivariate correlations (Pearson's $r>0.70$ ) among pairs of predictor variables. We subsequently conducted linear regression analysis to test each of the predictor variables against all the others and calculate tolerance and the variance inflation factor (VIF). Tolerance $>0.1$ and VIF $<10$ indicated lack of significant multicollinearity.

Statistical significance was set at $\mathrm{P}<0.05$. Statistical analyses were performed by the SPSS version 17.0 package (SPSS Inc., Chicago, IL, USA).

\section{Results}

Patient characteristics. A total of 76 patients developing PSA biochemical recurrence following radical prostatectomy 
Table I. Patient characteristics.

\begin{tabular}{|c|c|c|c|}
\hline Characteristics & Metastasis not confirmed & Metastasis confirmed & P-value \\
\hline Total number & 45 & 31 & \\
\hline Median age, years (range) & $68(51-81)$ & $65(47-85)$ & 0.251 \\
\hline \multicolumn{4}{|l|}{ pT staging, number (percentage) } \\
\hline IIB & $25(56)$ & $17(55)$ & \multirow[t]{2}{*}{0.846} \\
\hline III & $20(44)$ & $14(45)$ & \\
\hline Median Gleason score at diagnosis (range) & $6(4-9)$ & $7(4-9)$ & 0.006 \\
\hline $\begin{array}{l}\text { Median time to PSA recurrence } \\
\text { in months }(95 \% \mathrm{CI}) \text { (range) }\end{array}$ & $19(11-27)$ & $23(17-30)$ & 0.744 \\
\hline \multicolumn{4}{|l|}{$\begin{array}{l}\text { Additional diagnostic imaging modality } \\
\text { at biochemical recurrence, number } \\
\text { (row percentages) }\end{array}$} \\
\hline 18F-choline PET & $23(100)$ & $0(0)$ & \multirow[t]{2}{*}{$<0.001$} \\
\hline Magnetic resonance imaging & $3(15)$ & $17(85)$ & \\
\hline \multicolumn{4}{|l|}{ Dominant site of progression (percentage) } \\
\hline Bone & - & $15(48)$ & - \\
\hline Locoregional lymph nodes & - & $7(23)$ & - \\
\hline Distant lymph nodes & - & $5(16)$ & - \\
\hline Local progression & - & $4(13)$ & - \\
\hline
\end{tabular}

PSA, prostate-specific antigen; CI, confidence interval; PET, positron emission tomography.

Table II. Summary of median values for each test in patients with and without metastatic progression at the time of the most recent diagnostic imaging procedure.

\begin{tabular}{|c|c|c|c|c|c|}
\hline \multirow[t]{2}{*}{ Test } & \multicolumn{2}{|c|}{ Non-progressing } & \multicolumn{2}{|c|}{ Progressing } & \multirow{2}{*}{ P-value } \\
\hline & Median & Range & Median & Range & \\
\hline PSA (ng/ml) & 4.070 & $1.010-7.990$ & 5.660 & $1.040-14.520$ & 0.001 \\
\hline 2pPSA (pg/ml) & 7.51 & $1.470-15.590$ & 34.490 & $10.230-117.000$ & $<0.001$ \\
\hline fPSA (ng/ml) & 0.550 & $0.180-1.360$ & 1.460 & $0.280-3.280$ & $<0.001$ \\
\hline$\%$ fPSA & 15.066 & $8.480-34.380$ & 23.530 & $7.300-47.200$ & 0.001 \\
\hline PHI & 25.828 & $6.500-58.700$ & 56.490 & $26.670-539.870$ & $<0.001$ \\
\hline
\end{tabular}

PSA, prostate-specific antigen; 2pPSA, [-2]pro-PSA; fPSA, free PSA; PHI, prostate health index.

were studied. A total of $42(55 \%)$ and $32(45 \%)$ patients had stage IIB (pT2c, N0, M0) and stage III (pT3a, N0, M0) prostate cancer, respectively, at the time of surgery. During a median observation time of 26 months (range, 1-155) from surgery, a total of 31 patients had a diagnosis of metastatic progression, which was confirmed by diagnostic imaging studies. Table I shows the characteristics of these patients. Median age, stage at diagnosis of prostate cancer and time to biochemical recurrence were not different between the two groups. Patients with imaging-confirmed metastatic progression had a higher median Gleason score. Notably, 18F-choline PET was used only in patients with unconfirmed metastatic progression (negative CT scan of the chest and abdomen and negative bone scan). Finally, MRI was used more frequently in patients with confirmed metastatic progression, but in all cases it was prescribed following a positive bone scan or CT scan.

Median values in patients with and without metastatic progression. Median PSA, fPSA, \%fPSA, 2pPSA and PHI values and their ranges are reported in Table II for patients with and without metastatic progression. The values were statistically significantly higher in patients with metastases. However, 2pPSA was almost 5-fold higher in metastatic compared with non-metastatic patients. Of all the pairs of tests, only PSA and fPSA revealed a correlation suggesting possible multicollinearity (coefficient $0.77, \mathrm{P}<0.001$ ). A borderline correlation coefficient of 0.67 was also observed for 2pPSA and fPSA. Additionally, the correlation coefficient between PSA and 
Table III. Analysis of the predictive role of each test with respect to the likelihood of metastatic progression (results considered as continuous variables).

\begin{tabular}{|c|c|c|c|c|c|c|}
\hline \multirow[t]{2}{*}{ Test } & \multicolumn{2}{|c|}{ Each single test } & \multirow[b]{2}{*}{ P-value } & \multicolumn{2}{|c|}{ Multivariate analysis } & \multirow[b]{2}{*}{ P-value } \\
\hline & $\mathrm{OR}^{\mathrm{a}}$ & $95 \% \mathrm{CI}$ & & $\mathrm{OR}^{\mathrm{a}}$ & $95 \% \mathrm{CI}$ & \\
\hline PSA (ng/ml) & 1.716 & $1.236-2.384$ & 0.001 & 0.834 & $0.363-1.915$ & 0.668 \\
\hline 2pPSA (pg/ml) & 1.895 & $1.216-2.953$ & 0.005 & 1.959 & $1.204-3.186$ & 0.007 \\
\hline fPSA (ng/ml) & 1.411 & $1.190-1.673$ & $<0.001$ & & $\mathrm{NI}$ & \\
\hline$\%$ fPSA & 1.151 & $1.055-1.255$ & 0.001 & - & - & - \\
\hline PHI & 1.188 & $1.091-1.294$ & $<0.001$ & - & - & - \\
\hline
\end{tabular}

${ }^{\text {a}}$ Values corrected by stage at diagnosis (IIB vs. III) and Gleason score (continuous values). OR, odds ratio. Odds ratios represent the increase in the probability of metastasis by each unit increase in the value of the marker for PSA, 2pPSA, \%fPSA and PHI, and by each 0.1 unit increase in the value of fPSA. NI, not included in the multivariate model; CI, confidence interval; PSA, prostate-specific antigen; 2pPSA, [-2]pro-PSA; fPSA, free PSA; PHI, prostate health index.

Table IV. Sensitivity and specificity of cut-off values for each test with respect to the presence of metastatic disease.

\begin{tabular}{lrcc}
\hline Test & Cut-off & Sensitivity & Specificity \\
\hline PSA $(\mathrm{ng} / \mathrm{ml})$ & 4.360 & 0.77 & 0.60 \\
2pPSA $(\mathrm{pg} / \mathrm{ml})$ & 12.250 & 0.97 & 0.80 \\
fPSA $(\mathrm{ng} / \mathrm{ml})$ & 0.625 & 0.87 & 0.64 \\
\%fPSA & 17.836 & 0.81 & 0.60 \\
PHI & 31.142 & 0.97 & 0.69
\end{tabular}

PSA, prostate-specific antigen; 2pPSA, [-2]pro-PSA; fPSA, free PSA; PHI, prostate health index.

2pPSA was $0.40(\mathrm{P}<0.001)$, suggesting a lack of multicollinearity between the two variables. If fPSA was excluded from the list of variables, the calculation of tolerance and VIF confirmed a lack of significant multicollinearity.

Association between each test and the likelihood of metastatic progression as continuous variables. We subsequently studied the association between each test and the likelihood of metastatic progression by logistic regression analysis considering test results as continuous variables. After studying different multivariate models including either PSA or fPSA, but not both variables together, fPSA was excluded from multivariate analyses due to multicollinearity. The results of logistic regression analyses are shown in Table III. Although each single test correlated significantly with progression, only 2 pPSA remained statistically significant in a multivariate model including all the tests. Table III reports a multivariate analysis model where PSA and 2pPSA have been maintained to reveal the predictivity of these two tests.

Detection of metastatic disease using ROC curves and identification of cut-off values. ROC curves for the 5 tests are shown in Fig. 1. By analyzing the coordinates of the ROC curves we identified cut-off values for each test that were associated with the best performance in terms of sensitivity and specificity,

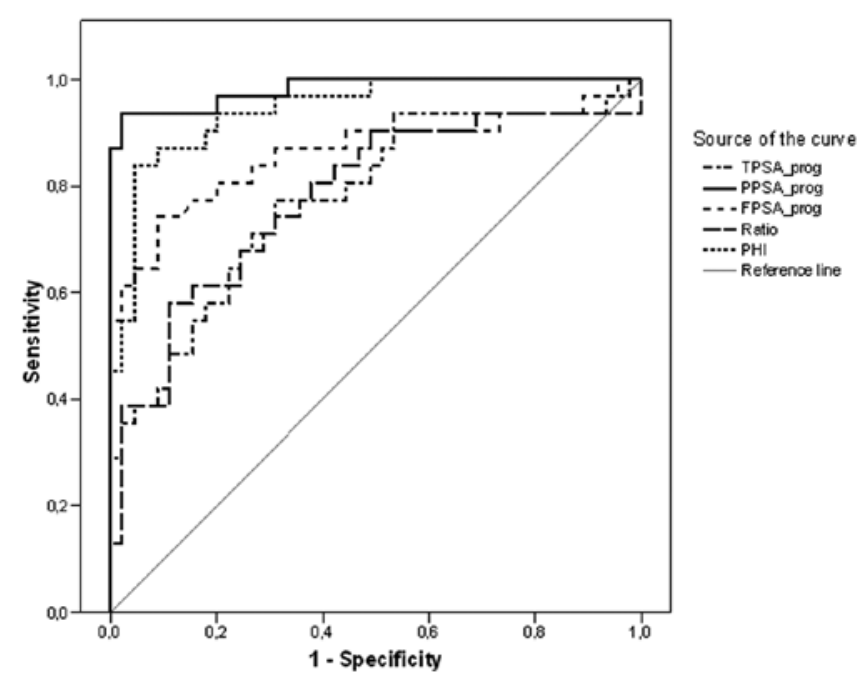

Figure 1. Receiver operating characteristics (ROC) analysis of the diagnostic performance of PSA, 2pPSA, fPSA, \% fPSA and PHI with respect to the detection of metastatic disease. PSA, prostate-specific antigen; 2pPSA, [-2]pro-PSA; fPSA, free PSA; PHI, prostate health index.

and results are reported in Table IV. Table V shows results of the analysis of the predictive value of each test, dichotomized around cut-off values identified by the ROC curves, with respect to metastatic progression. In the multivariate model including all the tests, only 2pPSA and PHI retained statistical significance.

\section{Discussion}

A persistently elevated or rising serum level of PSA following radical prostatectomy universally antedates metastatic progression and prostate cancer-specific mortality, but it is not a surrogate for these endpoints $(2,17)$. This condition indicates a heterogeneous patient population in which the prognosis is highly variable and the management of patients is controversial. New markers for risk stratification are therefore required.

In this small-scale study we found that 2pPSA has the potential to outperform serum PSA and other PSA-based tests 
Table V.Analysis of the predictive role of each test with respect to the likelihood of metastatic progression (results dichotomized around cut-off values identified by ROC analysis).

\begin{tabular}{|c|c|c|c|c|c|c|}
\hline \multirow[t]{2}{*}{ Test } & \multicolumn{2}{|c|}{ Each single test } & \multirow[b]{2}{*}{ P-value } & \multicolumn{2}{|c|}{ Multivariate analysis } & \multirow[b]{2}{*}{ P-value } \\
\hline & $\mathrm{OR}^{\mathrm{a}}$ & $95 \% \mathrm{CI}$ & & $\mathrm{OR}^{\mathrm{a}}$ & $95 \% \mathrm{CI}$ & \\
\hline PSA (ng/ml) & 5.317 & $1.743-16.223$ & 0.003 & - & - & - \\
\hline 2pPSA $(\mathrm{pg} / \mathrm{ml})$ & 118.172 & $13.041-1069.425$ & $<0.001$ & 56.411 & $5.944-535.361$ & $<0.001$ \\
\hline $\mathrm{fPSA}(\mathrm{ng} / \mathrm{ml})$ & 8.893 & $2.792-28.907$ & $<0.001$ & NI & & \\
\hline$\%$ fPSA & 4.997 & $1.655-15.089$ & 0.004 & - & - & - \\
\hline PHI & 57.886 & $6.792-480.632$ & $<0.001$ & 25.161 & $2.451-257.471$ & 0.006 \\
\hline
\end{tabular}

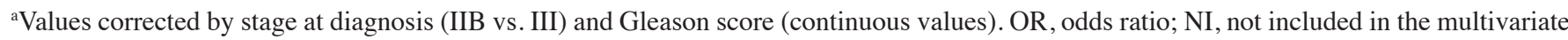
model; PSA, prostate-specific antigen; 2pPSA, [-2]pro-PSA; fPSA, free PSA; PHI, prostate health index.

in predicting metastatic prostate cancer. Notably, whereas all the tests considered correlated significantly with progression when values were studied as continuous variables, only 2 pPSA remained statistically significant in the multivariate analysis.

Median values were significantly higher in patients with confirmed recurrent disease for all the tests. However, the largest difference in median values between recurring and non-recurring patients was observed with 2pPSA, suggesting the potential for improved sensitivity and specificity. ROC analysis revealed that for 2pPSA a cut-off value was established that was associated with the highest sensitivity and specificity, compared with the other tests. When test results were dichotomized around cut-off values studied by ROC analysis, the multivariate analysis revealed that 2pPSA had the highest predictive value for the diagnosis of metastatic disease. The PHI index, which includes 2pPSA, also retained statistical significance, but its performance was inferior to that of 2pPSA.

A noteworthy finding of our study was that fPSA and \% fPSA were significantly higher in metastatic vs. nonmetastatic patients. fPSA is a circulating catalytically inactive form of PSA (18). In patients with increased PSA due to benign prostate disease, a high \%fPSA is associated with a significant reduction in false positive results and allows a reduction in unnecessary bioptic procedures (19). Conversely, a low \%fPSA predicts a positive finding at prostate biopsy across a wide range of increasing PSA values (20-23). Although fPSA and \%fPSA have been extensively studied in the setting of prostate cancer screening, their value in the metastatic setting has rarely been considered. A single study, published in 1998, failed to reveal a clear \%fPSA pattern in 52 males who had undergone radical prostatectomy and who subsequently developed biochemical recurrence (24). In contrast to that study, our series included 31 patients with diagnostic imaging-proven metastatic prostate cancer. We believe that the biological significance of our findings in prostatectomized patients developing a high burden of metastatic disease should be further investigated.

To the best of our knowledge, this is the first study identifying at a role of 2pPSA in this patient setting, and this is the major strength of the study. In addition, all the patients were followed at two institutions where urologists and oncologists are part of the same multidisciplinary treatment team. Efforts were made to standardize medical management and diagnostic procedures in patients at risk of developing recurrent disease. The cross-sectional design is the main limitation of this study. Although PSA was monitored serially, 2pPSA was assessed only in serum samples drawn at the time of biochemical recurrence. No information may therefore be derived from our data on the potential of 2 pPSA, measured at the time of biochemical recurrence in predicting subsequent clinical progression.

For example, an indicator that has been derived from serial PSA monitoring is PSA doubling time (PSADT), which is calculated as the number of months for a certain level of PSA to increase by a factor of two (25). PSADT, together with time to PSA recurrence, have been shown to be significant predictors of prostate cancer-specific mortality $(2,26)$. If our findings regarding sensitivity and specificity of 2pPSA are confirmed in other series, it would be of note to explore whether the kinetics of 2pPSA are likely to add prognostic information in patients with biochemical recurrence following prostate cancer surgery.

In conclusion, this is the first study revealing that 2pPSA values are markedly correlated with the likelihood of positive diagnostic imaging findings in previously resected prostate cancer patients with biochemical recurrence and may be useful in assessing the need for salvage therapy. Despite the limitations of our study, we believe that our findings should be further investigated in this clinical setting.

\section{References}

1. Makarov DV, Loeb S, Getzenberg RH and Partin AW: Biomarkers for prostate cancer. Annu Rev Med 60: 139-151, 2009.

2. Antonarakis ES, Chen Y, Elsamanoudi SI, Brassell SA, Da Rocha MV, Eisenberger MA and McLeod DG: Long-term overall survival and metastasis-free survival for men with prostate-specific antigen-recurrent prostate cancer after prostatectomy: analysis of the Center for Prostate Disease Research National Database. BJU Int 108: 378-385, 2011.

3. Scattoni V, Roscigno M, Raber M, et al: Multiple vesico-urethral biopsies following radical prostatectomy: the predictive roles of TRUS, DRE, PSA and the pathological stage. Eur Urol 44: 407-414, 2003.

4. Kane CJ, Amling CL, Johnstone PA, et al: Limited value of bone scintigraphy and computed tomography in assessing biochemical failure after radical prostatectomy. Urology 61: 607-611, 2003.

5. Kattan MW, Wheeler TM and Scardino PT: Postoperative nomogram for disease recurrence after radical prostatectomy for prostate cancer. J Clin Oncol 17: 1499-1507, 1999. 
6. Makarov DV, Isharwal S, Sokoll LJ, et al: Pro-prostate-specific antigen measurements in serum and tissue are associated with treatment necessity among men enrolled in expectant management for prostate cancer. Clin Cancer Res 15: 7316-7321, 2009.

7. Mikolajczyk SD, Marker KM, Millar LS, et al: A truncated precursor form of prostate-specific antigen is a more specific serum marker of prostate cancer. Cancer Res 61: 6958-6963, 2001

8. Mikolajczyk SD, Catalona WJ, Evans CL, et al: Proenzyme forms of prostate-specific antigen in serum improve the detection of prostate cancer. Clin Chem 50: 1017-1025, 2004.

9. Sokoll LJ, Wang Y, Feng Z, et al: [-2]proenzyme prostate specific antigen for prostate cancer detection: a national cancer institute early detection research network validation study. J Urol 180 539-543, 2008

10. Semjonow A, Kopke T, Eltze E, Pepping-Schefers B, Burgel H and Darte C: Pre-analytical in-vitro stability of [-2]proPSA in blood and serum. Clin Biochem 43: 926-928, 2010.

11. Catalona WJ, Bartsch G, Rittenhouse HG, et al: Serum pro prostate specific antigen improves cancer detection compared to free and complexed prostate specific antigen in men with prostate specific antigen 2 to $4 \mathrm{ng} / \mathrm{ml}$. J Urol 170: 2181-2185, 2003.

12. Stephan C, Kahrs AM, Cammann H, et al: A [-2]proPSA-based artificial neural network significantly improves differentiation between prostate cancer and benign prostatic diseases Prostate 69: 198-207, 2009.

13. Sokoll LJ, Sanda MG, Feng Z, et al: A prospective, multicenter, National Cancer Institute Early Detection Research Network study of [-2]proPSA: improving prostate cancer detection and correlating with cancer aggressiveness. Cancer Epidemiol Biomarkers Prev 19: 1193-1200, 2010.

14. Le BV, Griffin CR, Loeb S, Carvalhal GF, Kan D, Baumann NA and Catalona WJ: [-2]Proenzyme prostate specific antigen is more accurate than total and free prostate specific antigen in differentiating prostate cancer from benign disease in a prospective prostate cancer screening study. J Urol 183: 1355-1359, 2010.

15. Catalona WJ, Sanda MG, Wei JT, et al: [-2]ProPSA in combination with PSA and free-PSA, using the Beckman Coulter access immunoassay systems improves prostate cancer detection relative to PSA and free-PSA. A multi-center prospective clinical study. J Urol 183: e717, 2010.
16. Clarke NW: What does failure after surgery or radiation mean? Eur Urol Suppl 7: 410-415, 2008.

17. Simmons MN, Stephenson AJ and Klein EA: Natural history of biochemical recurrence after radical prostatectomy: risk assessment for secondary therapy. Eur Urol 51: 1175-1184, 2007.

18. Jansen FH, Roobol M, Jenster G, Schroder FH and Bangma CH: Screening for prostate cancer in 2008 II: the importance of molecular subforms of prostate-specific antigen and tissue kallikreins. Eur Urol 55: 563-574, 2009.

19. Stenman UH, Leinonen J, Zhang WM, Finne P and Wu P: The clinical importance of free prostate-specific antigen (PSA). Curr Opin Urol 8: 393-399, 1998

20. Catalona WJ, Smith DS, Wolfert RL, Wang TJ, Rittenhouse HG, Ratliff TL and Nadler RB: Evaluation of percentage of free serum prostate-specific antigen to improve specificity of prostate cancer screening. JAMA 274: 1214-1220, 1995.

21. Partin AW, Catalona WJ, Southwick PC, Subong EN, Gasior GH and Chan DW: Analysis of percent free prostate-specific antigen (PSA) for prostate cancer detection: influence of total PSA, prostate volume, and age. Urology 48: 55-61, 1996.

22. Finne P, Finne R, Bangma C, Hugosson J, Hakama M, Auvinen A and Stenman UH: Algorithms based on prostate-specific antigen (PSA), free PSA, digital rectal examination and prostate volume reduce false-positive PSA results in prostate cancer screening. Int J Cancer 111: 310-315, 2004.

23. Finne P, Auvinen A, Maattanen L, et al: Diagnostic value of free prostate-specific antigen among men with a prostate-specific antigen level of $<3.0$ microg per liter. Eur Urol 54: 362-370, 2008.

24. Lin DW, Noteboom JL, Blumenstein BA, Ellis WJ, Lange PH and Vessella RL: Serum percent free prostate-specific antigen in metastatic prostate cancer. Urology 52: 366-371, 1998.

25. Vickers AJ, Savage C, O'Brien MF and Lilja H: Systematic review of pretreatment prostate-specific antigen velocity and doubling time as predictors for prostate cancer. J Clin Oncol 27: 398-403, 2009

26. Denham JW, Steigler A, Wilcox C, et al: Time to biochemical failure and prostate-specific antigen doubling time as surrogates for prostate cancer-specific mortality: evidence from the TROG 96.01 randomised controlled trial. Lancet Oncol 9: 1058-1068, 2008. 